\title{
La desalinizadora de agua de mar en Playas de Rosarito. Un proyecto estratégico frente a la dependencia del Río Colorado y la escasez de agua en Baja California
} Desalinating Seawater at Rosarito Beaches, a Strategic Project in the Face of Dependence on the Colorado River and Baja California's Water Scarcity

Vicente SÁnchez Munguía*

\section{RESUMEN}

Frente a la escasez de agua que afecta a las ciudades costeras de Baja California, las autoridades estatales y federales optaron por la construcción de una planta desalinizadora de agua de mar en Rosarito, con la posibilidad de exportar una parte de la producción a San Diego. El proyecto fue aprobado en 2017 en medio de cuestionamientos y está detenido por falta de consensos sobre los términos de la concesión a una empresa privada. El objetivo de este artículo es mostrar la importancia regional y binacional del proyecto, destacando el carácter tecnocrático con el cual se concibió y se ha gestionado, a partir de un análisis del proceso de autorización y una revisión de la literatura sobre la tendencia global de la desalinización ante la escasez de agua. Se concluye que el proyecto se ha perfilado desde hace mucho tiempo y hay un gran interés a favor en México y Estados Unidos, lo cual le confiere mayor fuerza y probabilidad de realización en ausencia de otras propuestas en el lado mexicano.

Palabras clave: escasez de agua, desalinización, variabilidad climática, Río Colorado, Tijuana.

\section{ABSTRACT}

Given the water scarcity in Baja California's coastal cities, state and federal authorities opted to build a seawater desalinization plant in Rosarito, with the possibility of exporting part of the production to San Diego. The project was approved in 2017 amidst controversy and has been halted due to the lack of consensus about the licensing terms to a private company. The aim of this article is to show the project's regional and binational importance, underlining the technocratic way it was conceived and handled, based on an analysis of the authorization process and a review of the literature covering the global trend toward desalinization given water scarcity. The author concludes that the project has been underway and planned for a long time and has sparked great interest in both Mexico and the United States, which strengthens the probability of its coming to fruition in the absence of other proposals on the Mexican side of the border.

Key words: water scarcity, desalinization, climate variability, Colorado River, Tijuana.

\footnotetext{
* Departamento de Estudios de Administración Pública, El Colegio de la Frontera Norte, <vsanchez@colef.mx>.
} 


\section{INTRODUCCIÓN}

La creciente demanda de agua en los centros urbanos del país y los problemas de contaminación de fuentes superficiales, la sobreexplotación de acuíferos y las sequías prolongadas atribuidas a la variabilidad climática se han vuelto punto de partida en la planeación de la política y la gestión hídrica; también han sido clave en el desarrollo de propuestas para enfrentar la escasez de agua en regiones más vulnerables a ese tipo de riesgos. Es dentro de ese contexto que encaja y se explica la propuesta de una planta desalinizadora de agua de mar en Rosarito, Baja California, como respuesta a la escasez y demanda de agua de Tijuana y su área metropolitana que incluye a Playas de Rosarito. Pero la aprobación y autorización del proyecto por el gobierno del estado y el congreso local transcurrieron a través de un proceso político y legislativo atropellado, lo cual terminó por paralizar el inicio de la construcción de la planta como estaba programado. Sin embargo, el proyecto no ha sido cancelado y su construcción puede iniciar en cualquier momento, dependiendo de cómo se renegocien los términos de su financiamiento y operación a largo plazo.

Sobre ese tipo de proyectos es importante mencionar que, a pesar de que siguen siendo objeto de discusión y polémica debido a los impactos ambientales en que incurren (descarga de salmuera al mar y sus efectos en la biodiversidad marina, emisiones a la atmósfera por la alta demanda de energía que requieren, entre otros), en las dos últimas décadas se ha registrado una tendencia internacional en la que países localizados en regiones áridas con problemas de disponibilidad de agua dulce han optado por la construcción y operación de desalinizadoras de aguas salobres provenientes de acuíferos, y de agua de mar en los casos de países con litoral costero. Se trate de aguas salobres o marinas, las decisiones sobre el uso de las tecnologías de desalinización buscan enfrentar los problemas de escasez y atender la demanda urbana de agua, principalmente.

En México, desde 2012 y años posteriores las plantas desalinizadoras de agua de mar se incorporaron como proyectos prioritarios de infraestructura hidráulica de la Comisión Nacional del Agua para abastecer de agua potable a ciudades costeras del país (Conagua, 2012; 2015; 2017). Para efectos prácticos, el país se adhirió a la tendencia en el desarrollo de ese tipo de proyectos en regiones áridas con precipitaciones escasas y eventos de sequías prolongadas. Con anterioridad, empresas privadas del ramo turístico ya habían instalado plantas desalinizadoras para el abasto de sus complejos en Baja California Sur y Quintana Roo; pero la primera y única planta que había venido operando desde 2007 para el servicio municipal es la de Los Cabos (Pombo et al., 2008; McEvoy, 2017). En Hermosillo, en el periodo de gobierno de Armando López Nogales (1997-2003), se intentó sin éxito la construcción de una planta de este 
tipo para abastecer la ciudad. El proceso llegó hasta la etapa de licitación del proyecto pero, según el exgobernador, la falta de apoyo del gobierno federal por el presidente Fox y la falta de interés del alcalde del Hermosillo surgido del Partido Acción Nacional imposibilitaron que el proyecto siguiera adelante y se lograra la construcción (López, 2019a); es decir, el proyecto no procedió debido al alto costo que el agua de la desalinizadora implicaba para la población, en tanto podía conseguirse de manera más económica en la zona agrícola de la costa (López, 2019b).

En las pasadas administraciones federales se promovieron y autorizaron nuevos proyectos de desalinización para atender la demanda en las ciudades del noroeste de México, con lo cual se perfilaron los proyectos de Guaymas-San Carlos y Puerto Peñasco en Sonora, Ensenada y Rosarito en Baja California, y una nueva planta en Los Cabos, Baja California Sur. En 2018, se puso en operación la planta desalinizadora de Ensenada, la primera de las que se previó construir en las costas del Pacifico noroeste del país por las administraciones federales pasadas. Por su parte, el gobierno de Baja California puso en operación en 2016 una planta de cinco litros por segundo (LPS) en Isla de Cedros e inició la construcción de otra planta en San Quintín, ambas en el municipio de Ensenada, con propósito de satisfacer la demanda local de agua para uso doméstico, aunque también es importante mencionar que en el Valle de San Quintín han venido operando varias plantas desalinizadoras para abastecer de agua a la actividad agrícola de exportación que se ha desarrollado en esa parte del estado de Baja California (Pombo, 2015).

En este trabajo se aborda el tema de la construcción de la planta desalinizadora de Rosarito, Baja California, partiendo de la consideración de que el proyecto fue concebido como la solución a la escasez de agua en el contexto de vulnerabilidad del estado frente a los eventos de sequía y la variabilidad climática que afectan la cuenca del río Colorado, la principal fuente de agua disponible para la región. Se trata de una cuestión de seguridad hídrica, pero la aprobación del proyecto ha tenido lugar en un contexto de desconfianza política hacia el gobierno del estado encabezado por Francisco Vega, a quien se atribuían conflictos de interés en torno a la concesión, además de que hubo resistencias debido a la carga financiera por las garantías de pago por parte de los organismos estatales encargados del agua y el gobierno del estado.

Analizado desde una perspectiva teórica, el proyecto de desalinización aprobado para Rosarito y Tijuana se vincula en su concepción al concepto de consenso tecnogenerancial acuñado por Swyngedouw y Williams (2016) y aplicado a procesos despolitizados según los cuales se conducen los proyectos basados en la tecnología a partir de una visión tecnocrática que busca evitar el debate y la politización en torno a las implicaciones de tales desarrollos. En el mismo sentido, McEvoy (2017) usa el concepto de sistema sociotécnico para identificar este tipo de proyectos y la necesidad de 
regular su desarrollo y operación, fortaleciendo primero las instituciones vinculadas a la planeación y operación de tales sistemas para inhibir eventos de corrupción, sobre todo en países de bajo desarrollo institucional.

Aquí se parte del supuesto de que el gobierno siguió un libreto tecnocrático sin contar con el consenso tecnogerencial y una base social para blindar el proyecto de la politización y disensos públicos. La discusión pública se ha enfocado en los términos de la concesión, las implicaciones financieras para el gobierno y la opacidad en torno al proyecto, dejando de lado las implicaciones de la desalinización como alternativa y la cooperación con San Diego como posibilidad. La construcción de la desalinizadora se ha convertido en un proyecto estratégico y prioritario para Baja California, tanto por su propia condición de disponibilidad de agua, como por el hecho de que es la opción (única) considerada por el gobierno del estado para garantizar el abasto de agua a la población de la zona costa.

El objetivo de este trabajo es visibilizar el carácter estratégico del proyecto de desalinización a la luz del desarrollo de la industria de este tipo de proyectos en las regiones áridas del mundo y las condiciones particulares de Baja California, pero también en el contexto de una conducción política caracterizada por la falta de transparencia y una inadecuada gestión del agua, la cual se ve primordialmente como fuente de ganancia económica. El artículo se basa en una amplia revisión de literatura sobre seguimiento en medios del proceso de aprobación del proyecto.

\section{LA DESALINIZACIÓN EN AUGE Y LAS CAUTELAS NECESARIAS}

Es un hecho que la desalinización de agua como alternativa a una demanda urbana creciente (Arahuetes y Villar, 2017) y la escasez provocada por las sequías prolongadas ya no es una novedad. Se trata de una solución técnica por la cual los gobiernos han optado con mayor frecuencia para enfrentar los problemas de escasez, pero no deja de provocar inquietudes, preocupaciones, debates y opiniones divergentes (Morote et al., 2017), muchas aún relacionadas con los impactos al medio ambiente (Tal, 2017), sea por el agua de rechazo y su descarga al mar con riesgos para la biodiversidad marina o por las emisiones a la atmósfera debido al alto consumo de energía, principalmente de origen fósil. En cuanto a las descargas al mar, las críticas se refieren al agua hipersalina (brine) con alta concentración de sal y residuos químicos usados en el proceso de producción, sobre todo en el eslabón de los microorganismos sensibles la alta concentración salina (Meerganz von Medeazza, 2005; García et al., 2007; Lattemann y Hopner, 2008; Drami et al., 2011), aunque se menciona la ausencia hasta ahora de estudios sistemáticos in situ para evaluar los impactos de ese tipo de vertidos al mar (García et al., 2007). 
Sin embargo, los estudios in situ que se han realizado han confirmado que la descarga de aguas de alta concentración de sal y residuos químicos afecta la flora marina y microorganismos como el fitoplancton, sobre todo cuando no hay un diseño adecuado del efluente de descarga con los dispositivos de dispersión para evitar o minimizar el impacto en el área en que se desechan las aguas de rechazo (Loya-Fernandez et al., 2012; Latterman et al., 2010; Dawoud y Mullá, 2012), los cuales aún siguen siendo motivo de preocupación tanto en la construcción, como en la operación de las plantas desalinizadoras. Es decir, no hay evidencias que muestren ausencia de impactos en el ambiente marino a partir de este tipo de descargas. En razón de estos señalamientos es que se plantea que una planta desalinizadora de agua debería cumplir con las regulaciones ambientales y ser rentable en su construcción, operación y gestión, tanto como en los costos asociados y el monitoreo (Berktay, 2011).

Se ha tendido a destacar las mejoras en la tecnología y de minimizar los impactos en aspectos específicos dentro del proceso de tratamiento, sea como medidas precautorias o como remediaciones, sea que se trate de regulaciones institucionales emitidas por los gobiernos para tratar de prevenir, minimizar o remediar los impactos en los distintos ambientes marinos, terrestres y atmosféricos. Por ejemplo, en Israel, ampliamente reconocido como país pionero en el uso intensivo de esta tecnología y con una de las plantas de este tipo más grandes del mundo, se ha señalado que hasta ahora no se cuenta con evidencias de impactos que afecten las zonas marinas de descarga y difusión de la salmuera o agua de rechazo (Tal, 2017).

En favor del uso de las tecnologías de desalinización se argumenta que se han vuelto mucho más eficientes en cuanto al consumo de energía y tienen la ventaja garantizar el acceso al agua sin depender del ciclo hidrológico (El Saliby et al., 2008), además de que son cada vez más asequibles desde el punto de vista del precio y con una tendencia hacia menores costos (Voutchkov, 2016), aunque es de destacar que el precio al consumidor por metro cúbico de agua desalinizada es muy variable de un país a otro y depende mucho de los estándares que deben cumplir los procesos de tratamiento en relación con medidas preventivas sobre su impacto al medio ambiente y el tipo de energía que usan. En el Mediterráneo español, la tarifa contratada inicialmente estaba entre 0.30 y 0.36 dólares estadunidenses por metro cúbico, en tanto que en las Islas Canarias estaba en un rango de 1.5 a 2 dólares el metro cúbico; en Israel, la tarifa oscila entre 0.95 y 0.89 dólares por metro cúbico; en Argelia, entre 0.57 y 0.78; en Asia van de 0.36 dólares en Singapur, a 1.03 dólares en India; en Australia, 0.89 dólares, y en California, entre 3 y 4 (Cosín, 2019).

Al considerar que el 40 por ciento de la población mundial se localiza en las inmediaciones de las zonas costeras (De Andrés y Barragán, 2016), la desalinización de agua del mar es una alternativa que ha logrado aceptación, la cual se expresa en el 
creciente número de plantas desalinizadoras que operan en los litorales de diversas regiones del planeta, lo que ha llevado a cuadruplicar el volumen de agua producida en las últimas décadas, principalmente en el Medio Oriente, en el Mediterráneo europeo, Australia y en Estados Unidos ${ }^{1}$ (García et al., 2007; Drami et al., 2011; Lattemann y Hopner, 2008).

La tecnología de ósmosis inversa es la más expandida para desalinizar agua y se presume que ha ganado terreno en preferencias por los avances en su eficiencia energética y en costos de producción. Cabe destacar que la desalinización concebida como solución a la escasez de agua deriva de ser una fuente no disputada y que se ha conducido en un marco de arreglos de tipo tecnogerencial (Swyngedow y Williams, 2016) socialmente asimilados, que incluyen las asociaciones público-privadas (APP) como parte de los planes de negocio, sobre todo cuando ha habido mucha crítica y resistencia activa a la gestión privada de los servicios de agua en todas partes; ésta sería una forma de evadir tales resistencias a la participación privada en el sector. Desde la década de 1980 la desalinización, en sus diversas variantes tecnológicas, se ha desarrollado bajo una perspectiva empresarial en todo el mundo (Nair y Kumar, 2013).

Información de la International Desalination Association (IDA) indica que actualmente operan más de veinte mil plantas desalinizadoras en todo el mundo, distribuidas en más de cien países, principalmente en países del Medio Oriente como Arabia Saudita, ${ }^{2}$ Emiratos Árabes Unidos e Israel; ${ }^{3}$ en el norte de África destacan Libia y Argelia; en el continente europeo, España ${ }^{4}$ es el principal país usuario de las tecnologías de desalinización de agua de mar. Este país optó por la desalinización en ciudades del Mediterráneo ante la imposibilidad política de realizar trasvases del norte al sureste o levante, aunque desde la década de 1960 desaliniza agua de mar para uso doméstico en las Islas Canarias (García-Rubio y Guardiola, 2012). A su vez, Australia desarrolló un conjunto de plantas desalinizadoras para enfrentar problemas de escasez debido a las sequías prolongadas que afectaban a sus principales ciudades. Estados Unidos, por su parte, se ha convertido es el principal país con plantas desaladoras de aguas salobres o brackish water extraídas del subsuelo; algunas operan en ciudades fronterizas con México, como Donna y El Paso en el estado de Texas. En 2015, se puso en

\footnotetext{
${ }^{1}$ En este país, el agua desalada proviene principalmente del subsuelo (brakish water); hay oposición activa del movimiento ambientalista a las plantas desalinizadoras de agua de mar, además de regulaciones muy estrictas para su operación.

${ }^{2}$ En Arabia Saudita, cuatro de cada cinco litros de agua que consume son producidos por desaladoras, es decir, el 80 por ciento del agua que consume su población.

${ }^{3}$ Estos países tienen las plantas desalinizadoras más grande del mundo por su capacidad instalada para la producción de agua por día.

${ }^{4}$ La planta desalinizadora más grande de España se encuentra en Torrevieja, Alicante; no está en operación aún y proyecta producir doscientos cuarenta mil metros cúbicos por día, la mitad para usos urbanos y la otra mitad para usos agrícolas en Murcia y Alicante.
} 
operación la planta desaladora en San Diego, California, la más grande de su tipo en el continente por su capacidad de producción (Fikes, 2015).

Desde una perspectiva más amplia, los impactos ambientales incluyen desde el proceso de construcción de la planta y el entorno de su localización, hasta el movimiento de materiales y ruidos cuando se localizan en las inmediaciones urbanas, así como sus afectaciones al paisaje (Hopner y Wildenberg, 1996), al igual que efectos de la operación de la planta, cuyo proceso de producción implica una demanda considerable de energía, por lo general de origen fósil que se traduce en altas emisiones a la atmósfera de gases de efecto invernadero (greenhouse gas), con impacto en el calentamiento de la atmósfera (Lattemann y Hopner, 2008).

Las evaluaciones de impacto de la desalinización en regiones como el Mediterráneo para el caso de Israel y España, y el Mar Rojo, el Golfo Pérsico y Australia, sugieren que lo más importante es que se atienda de manera prioritaria la construcción o reforzamiento de los mecanismos institucionales de carácter regulatorio para gestionar la infraestructura tecnológica y sus resultados, sobre todo en países donde la institucionalidad es débil (McEvoy, 2017). Lo más importante es el desarrollo de capacidades institucionales, lo mismo en las empresas locales de agua (organismos operadores) como en las oficinas de planeación urbana, puesto que la debilidad institucional hace que esos organismos sean susceptibles a la corrupción (McEvoy, 2017; Morote et al., 2017).

\section{La dependencia de Tijuana del agua del Río Colorado}

Puede asumirse que el proyecto de una planta desalinizadora para Tijuana y su entorno metropolitano es parte de una política de seguridad hídrica frente a la escasez y los pronósticos que implican reducciones en la disponibilidad de agua en la cuenca baja del Río Colorado. La importancia estratégica del proyecto sólo se entiende a partir de la información sobre las características hidrológicas del estado de Baja California, donde el Río Colorado destaca como principal fuente de provisión de agua para Tijuana y su área metropolitana, e incluso Ensenada, lo mismo que para el Valle de Mexicali como espacio territorial en el que se concentra abrumadoramente la mayor proporción de agua disponible en el estado, tanto de origen superficial como subterránea.

La información oficial señala que Baja California es un estado con precipitaciones pluviales que van de 200 a 300 mm (milímetros) al año en una pequeña porción de su territorio, mientras en el resto del estado llegan a $50 \mathrm{~mm}$ (GBC, s. f). Se trata de un territorio con muy baja disponibilidad hídrica, la cual asciende a $3250 \mathrm{Mm}^{3}$ (millones de metros cúbicos) y, de éstos, el 88 por ciento se concentra en el Valle de Mexicali, 
cantidad de la cual el Río Colorado aporta el 57 por ciento, ${ }^{5}$ es decir, $1681.5 \mathrm{Mm}^{3}$ (GBC, s. f.); el resto son aguas subterráneas que provienen de un acuífero reportado por la Comisión Nacional del Agua (Conagua) como sobreexplotado.

En lo que se refiere a Tijuana, el acceso al agua ha sido desde siempre un importante desafío para las autoridades, y por lo menos desde la década de 1960 ya se presentaba como un problema complejo, lo que las llevó a en ese entonces a buscar alternativas para atender la demanda en constante crecimiento (Bustamante, 1999), debido a su dinámica demográfica y el inicio de los programas de industrialización promovidos por el gobierno federal. Ante la insuficiencia de las fuentes locales para cubrir las necesidades de la población, el gobierno del estado, con el apoyo de la federación, inició la construcción del acueducto Río Colorado-Tijuana para transportar el líquido desde el Valle de Mexicali hasta la costa para satisfacer la demanda de la población y la actividad económica de Tecate, Tijuana, durante las siguientes décadas (Rosarito todavía era parte del municipio de Tijuana). El acueducto se empezó a construir en 1975 y empezó a operar en 1982 (CEA, s. f.), justo cuando ya había reclamos y movilizaciones sociales por la carencia de agua en distintas zonas de la ciudad (Negrete, 1990).

Desde que se puso en operación, el acueducto ha sido, hasta la actualidad, la solución a la falta de agua en Tijuana y su entorno metropolitano, pero esta conexión también dio pie a la dependencia de la zona costa del agua del Río Colorado ante la limitada captación que logra la presa "Abelardo L. Rodríguez" dado que, como se ha señalado, se localiza en una subregión caracterizada por un régimen de bajas precipitaciones, en tanto que el acuífero subyacente al Río Tijuana ha perdido importancia debido a la contaminación por vertido de aguas residuales de origen industrial, la pobre infraestructura de drenaje y el descuido de las autoridades de los tres niveles de gobierno. Según la Comisión Estatal del Agua (CEA, 2015), los cuatrocientos cincuenta litros por segundo que están en capacidad de producir requieren tratamiento para separar el hierro y el manganeso que contienen; adicionalmente, se ha perdido capacidad de infiltración al haberse canalizado con concreto los cauces del Río Tijuana y del arroyo Alamar en sus trayectos urbanos, además de que los pozos del acuífero de Rosarito contienen intrusión salina debido a la sobreexplotación de que han sido objeto (CEA, 2015).

En la actualidad, Tijuana y su área metropolitana dependen casi en su totalidad del agua que transporta el acueducto desde el Valle de Mexicali; las fuentes locales, tanto superficiales como subterráneas de agua, juegan un rol marginal, su aporte a las redes de distribución en los últimos años fluctúa entre el 1 y el 5 por ciento. De acuerdo con la Comisión Estatal de Servicios Públicos de Tijuana (CESPT) (2017), ya en

\footnotetext{
${ }^{5} \mathrm{El}$ aporte de 1850 millones de metros cúbicos que se reciben en cumplimiento del tratado internacional de 1944 se comparte con Sonora (San Luis Río Colorado).
} 
2014 el 99 por ciento del agua que distribuyó esta comisión provino del Río Colorado y las condiciones no han cambiado. Esta información permite visibilizar también la vulnerabilidad de la zona costa del estado frente a los eventos de sequías y cualquier otro tipo de situación que pudiera afectar la continuidad en la operación del mencionado acueducto; pero, al mismo tiempo, confirma la dependencia que se estableció entre la zona costa de Baja California del trasvase de agua desde el Valle de Mexicali. Por lo tanto, Tijuana y su área metropolitana están fuertemente vinculadas a las decisiones y acciones de política relacionadas con la gestión de la escasez y los efectos de la variabilidad climática en esta cuenca internacional.

\section{Pasado y futuro de desalinización de agua en Rosarito}

Las decisiones que se han tomado en relación con la construcción de la planta desalinizadora en Rosarito no son ajenas a lo que ocurre en la cuenca del Río Colorado y la forma en que las autoridades de los dos países han acordado para enfrentar las condiciones adversas en cuanto a la disponibilidad de agua para usos público y urbano que incluyen a la población de la zona costa. Las actas 319 y 323 de la Comisión de Límites y Aguas (CILA) no dejan lugar a dudas; en la primera de estas actas se estableció con claridad el acuerdo para construir dos plantas desalinizadoras en la región (Sánchez y Cortez, 2015; Wilder et al., 2016; Mumme et al., 2017). Con sustento en la segunda de estas actas, el presidente de Estados Unidos decretó en agosto de 2019 la contingencia por sequía en la cuenca del Colorado, tomando en cuenta la baja en el caudal que conduce este río internacional y el nivel de agua en el lago Mead (Presa Hoover) en Estados Unidos, como lo establecen las previsiones del Acta 323 de la cILA, lo que obliga a México a un ahorro de agua por cincuenta y un millones de metros cúbicos en el lago Mead, siguiendo la tabla de ahorros acordada en esa acta.

El tema de la desalinización de agua en Rosarito ha estado presente desde hace tiempo, por lo menos en el plano conceptual, y se ha mencionado como una expresión de la cooperación hídrica binacional para beneficio de Tijuana y San Diego (Castro y Sánchez, 2008); como se dijo antes, tanto la Conagua en su portafolio de proyectos prioritarios de las administraciones anteriores, como la misma ciLA en el Acta 319, dieron sentido de realidad a la concreción de proyectos de desalinización en esta parte del país, en respuesta a los problemas que seguridad hídrica relacionados con los cambios en el balance hídrico de la cuenca baja del Río Colorado. La crítica y oposición al proyecto se ha enfocado en la forma en que el gobierno del estado y la Conagua gestionaron el proceso político para la aprobación de la desalinizadora, así como la falta de transparencia sobre la concesión de un contrato que se percibe ventajoso 
para la empresa involucrada, sobre todo por la carga para las finanzas estatales que traería a los consumidores, pero también por supuestos o reales conflictos de interés de funcionarios del gobierno. Es decir, los cuestionamientos han sido falta de transparencia en la aprobación de la concesión y no sobre el proyecto como tal, con sus ventajas e implicaciones ambientales de diverso orden.

Por otro lado, la desalinización no es un tema nuevo o ajeno en esta región. Una mirada a los antecedentes y experiencias de desalinización de agua de mar en Baja California conduce a Rosarito, el lugar donde ahora se busca construir el nuevo proyecto y donde ya en la década de 1960 la desalinización para producir agua potable estuvo entre las alternativas propuestas para el abasto regional. En este sentido, el excomisionado de la CILA, Joaquín Bustamante (1999: 404) refiere al respecto que "Se construyó y se puso en servicio la planta desaladora en Rosarito, pero en su operación se presentaron serios y muy frecuentes problemas que durante varios años de servicio demostraron que la planta no constituía una solución económica, práctica o confiable al problema de falta de agua potable para la ciudad".

Otro autor señala que "en el año de 1970 se puso en operación en la Planta Termoeléctrica de Rosarito, la primera planta desaladora de México, que fue en su tiempo una de las más grandes del mundo, con una capacidad de producción de 320 LPS, 28350 metros cúbicos por día" (Correa, 2007: 5). Este mismo autor señala que en 2003 el gobierno del estado abortó la propuesta de una empresa gasera internacional para construir un "centro regional de energía" que incluía una planta desalinizadora en Rosarito con capacidad de 859 LPS (Correa, 2007: 25). Como puede verse, en este último caso también los intereses del gobernador en turno jugaron a favor de una empresa que al final concretó el proyecto de la estación de regasificación en las proximidades de Ensenada, con lo cual la propuesta del "centro de energía", que incluía la producción de agua mediante tecnología de membranas, se quedó en el camino.

En resumen, la escasa disponibilidad de agua dulce por la baja precipitación y la creciente demanda en la región han convertido a la desalinización de agua en una alternativa y, en ese sentido, ha sido un tema latente desde hace tiempo y que nunca se ha descartado del todo en esta parte del país. Ahora, con esta tendencia en la aplicación de este tipo de soluciones tecnológicas y las posibilidades negocio en este campo, la realización de este proyecto empata con cierta urgencia por encontrar soluciones a los problemas de escasez de agua en Tijuana y Rosarito. Sin embargo, es notable que, teniendo la Conagua como eje de su política la gestión integrada de recursos hídricos, no se haya hecho una sola mención de otras medidas para mejorar la eficiencia en la gestión del agua disponible u otras alternativas al desarrollo de este proyecto; nada que se refiera al tratamiento de las aguas residuales, su reuso e incluso su potabilización 
como se hace ya en otras ciudades del mundo ${ }^{6}$ en lo que se conoce como economía circular del agua, a pesar del problema internacional por el vertido de aguas residuales sin tratamiento que van de Tijuana a las playas de San Diego. ${ }^{7}$

En lo que se refiere a la propuesta en torno al proyecto autorizado en 2017, Mumme et al. (2017) comentan que desde 2007 se había proyectado la idea de una planta de desalinización en Rosarito con vínculos hacia el este de San Diego, es decir, con el propósito de exportar agua hacia el distrito de Otay Mesa, y habría llamado la atención de diversos agentes en Estados Unidos interesados en que se liberara agua para usos ambientales y la restauración del delta del Colorado. En 2012, el Otay Water District habría logrado un punto de acuerdo con la empresa NSC mexicana filial de Consolidated Water Company para la compra de cuarenta millones de galones por día, generados a partir de la desalinizadora en Rosarito (Mumme et al., 2017).

El Acta 319 de la CILA, firmada en 2012, incluyó la construcción de dos plantas desalinizadoras entre las medidas de solución ante eventuales reducciones en el caudal disponible del río (CILA, 2012). La misma acta se refiere a proyectos de conservación de agua en zonas agrícolas, de conservación y reuso en zonas urbanas, así como nuevas fuentes de agua, entre las cuales se incluyen las plantas desalinizadoras en el Océano Pacífico y en el Mar de Cortés (Sánchez y Cortez, 2015). Está claro que la opción de un proyecto de desalinización de agua ha sido definida en el marco de un acuerdo binacional que forma parte del tratado internacional que regula la distribución de agua de esta cuenca, aunque el mismo no determine en forma puntual y específica el lugar exacto de su localización, lo cual se entiende que se trata de un asunto interno a decidir por el gobierno de México y las autoridades estatales de Baja California. En el acta mencionada tampoco se hace referencia a la exportación de agua, pero sí al propósito de prevenir impactos por los cambios que afectan la disponibilidad en la cuenca baja del Río Colorado y de caudales para usos ambientales en el delta de éste. Sin embargo, de acuerdo con lo expuesto por Mumme et al. (2017), se infiere que las decisiones del gobierno y del congreso estatal no han sido ajenas a los arreglos e intereses en juego en el negocio del agua que ya se había convenido entre el Otay Water District y NSC.

Al respecto, la información de la empresa Consolidated Water, en su portal institucional, refiere que:

\footnotetext{
${ }^{6}$ Notablemente Windhoek, capital de Namibia, desde 1968, y Singapur en años más recientes (Hartley et al., 2019), pero también en San Diego con el Pure Water Program (City of San Diego, s. f.).

${ }^{7}$ El vertido recurrente de aguas residuales sin tratamiento que ha estado cruzando de Tijuana a San Diego en los últimos años ha llevado a los gobiernos a la firma del Acta 320 de la cILA para resolver el problema, provocado por la deficiente infraestructura de drenaje y la obsolescencia de las plantas de tratamiento de Tijuana, donde el agua residual no se ve aún como recurso disponible y aprovechable por las autoridades mexicanas.
} 
En mayo de 2010, adquirimos una participación del 50 por ciento en NSC Agua, S.A. de C.V. ["NSC"], una empresa mexicana en etapa de desarrollo. Desde entonces, hemos comprado, a través de la conversión de un préstamo que hicimos a NSC, suficientes acciones para elevar nuestra participación en NSC al 99.9 por ciento. NSC se formó para perseguir un proyecto [el "Proyecto"] que abarca la construcción, operación y propiedad minoritaria de una planta de desalinización por ósmosis inversa de agua de mar de cien millones de galones por día que se ubicará en el norte de Baja California, México, y acueductos acompañantes para entregar agua al sistema mexicano de agua potable. Creemos que el Proyecto puede tener éxito debido a la creciente necesidad de un nuevo suministro de agua potable para el área del norte de Baja California, México (Consolidated Water, 2015).

Igualmente, la aprobación de la Ley estatal de Asociaciones Público-Privadas en agosto de 2014, sin oposición política alguna, se había perfilado en relación con el proyecto de la planta desalinizadora en Rosarito y la concesión a una empresa que ya había logrado algún tipo de acuerdo y, por lo tanto, adelantar en el diseño del proyecto de acuerdo con los términos de referencia que se iban a solicitar. Por la forma en que se habían aprobado las propuestas del gobierno, éste no previó que fuera a tener que enfrentar mayor oposición.

El 23 de septiembre de 2016, el gobernador del estado firmó el contrato con la empresa concesionaria y en diciembre del mismo año el congreso del estado aprobó una nueva legislación estatal con el propósito de regular los servicios de agua con una visión privatista que implicaba una subida considerable de las tarifas como forma de garantizar el pago a la empresa concesionaria, lo cual motivó movilizaciones sociales en su contra.

El rechazo social a la nueva ley estatal de aguas obligó al gobierno a abrogarla y buscar otra estrategia política para aprobar el esquema legal bajo el cual el organismo operador de Tijuana y el gobierno del estado debían garantizar el pago ${ }^{8}$ del agua producida por NSC en la planta desalinizadora de Rosarito durante treinta y siete años. El Decreto 57 del congreso local que establecía el programa de pagos fue ampliamente cuestionado y motivó recursos judiciales ante la Suprema Corte de Justicia de la Nación por presuntas irregularidades del proceso legislativo (Corpus, 2018).

Desde la perspectiva gubernamental, el proyecto se justifica a partir de la limitada disponibilidad de agua de las fuentes tradicionales en la zona costa del estado y las complicaciones para lograr contratos de largo plazo con los agricultores para la

\footnotetext{
${ }^{8}$ El monto autorizado por el congreso del estado para pago de agua a la empresa desalinizadora es por 149312018 pesos mensuales durante treinta y siete años de la concesión, lo que se traduce en poco más de 67000000000 de pesos.
} 
transferencia temporal de los derechos de agua para Tijuana y Rosarito, en tanto que la desalinización representa "el aprovechamiento de una fuente inagotable de agua" (CEA, 2015). Además, se señala también que, al abastecer de agua a Tijuana y Rosarito con la producción de la planta desalinizadora, se busca restablecer el equilibrio del acuífero de Mexicali. Por lo que se refiere al esquema de inversión mayormente privada para la construcción de la desalinizadora, se hace referencia a la ventaja que representan las condiciones técnicas de su realización y el logro de resultados en menor tiempo (CEA, 2015) y, en cuanto al costo del agua, el ejercicio de la CEA señala que el más bajo corresponde a la desalinización, el cual asciende a 16.61 pesos por metro cúbico.

La perspectiva del gobierno del estado y la Conagua quedó claramente establecida por el propio gobernador, al señalar ante los medios que el proyecto de desalinización bajo el esquema de inversión público-privada era la mejor opción que se había encontrado para solucionar el problema de escasez de agua en Tijuana: "Hace más de tres años, como gobernador, asistí con la autoridad reguladora de agua que es la Comisión Nacional del Agua; ahí nos sentamos a ver de qué forma resolvemos el problema de escasez de agua en Baja California. Trabajamos por más de dos años, llegando a la conclusión de que la forma más eficiente, más efectiva y más sustentable económicamente era a través de inversión público-privada" (Arellano Sarmiento, 2017). En este sentido, es pertinente señalar que la tendencia internacional en el desarrollo de proyectos de desalinización muestra que se trata de un campo dominado por contratos de inversión público-privada bajo concesiones para la construcciónoperación e incluso la distribución y cobranza, en una versión del mercado privado de agua, en una nueva fuente que no está sujeta a la disputa de derechos, como ocurre con las fuentes tradicionales.

En cuanto a las manifestaciones contrarias al proyecto, se puede observar que la resistencia y rechazo fueron propiciados por el mismo gobierno en la medida en que se aisló de un conjunto de actores sociales y políticos en el estado, ${ }^{9} \sin$ lograr un consenso político y social suficiente en apoyo del proyecto. En ese momento, el gobierno del estado ya era abiertamente señalado por la opinión pública a causa de conflictos de interés que favorecían los negocios de cercanos al entorno de los funcionarios involucrados en las decisiones gubernamentales, sobre todo con empresas vinculadas a este proyecto. ${ }^{10}$

\footnotetext{
${ }^{9}$ Eso explica que incluso personalidades reconocidas dentro del propio panismo bajacaliforniano se hayan manifestado contra el proyecto y en la votación dentro del congreso hubiera oposición del propio partido en el gobierno.

${ }^{10}$ Algunas de las críticas se refieren a vínculos directos de funcionarios del gobierno con empresas constructoras y proveedoras de servicios incluidos en el proyecto.
} 
Swyngedouw y Williams (2016) han argumentado que, aunque los proyectos de desalinización son soluciones de corte tecnocrático, tienden a ser presentados como incontestables, aisladas de los procesos sociopolíticos que implican el conflicto, sin evitar la politización. Se trata de propuestas tecnocráticas que se presentan al amparo de consensos tecnogerenciales construidos en torno a las soluciones técnicas aisladas de los escenarios políticos. El proceso que siguió el proyecto de desalinización en Rosarito responde a ese perfil tecnogerencial, pero el gobierno del estado lo condujo de acuerdo con sus propios intereses y excluyó a otros actores, sin lograr el suficiente consenso político regional en torno a la propuesta. ${ }^{11}$

El proyecto se aprobó en medio de cuestionamientos al proceso, y las implicaciones financieras fueron consideradas onerosas para la hacienda pública estatal. Las críticas han venido de todos lados, incluidas las del exgobernador José Guadalupe Osuna Millán, con reconocida experiencia en la gestión de organismos de agua y con una posición crítica a la propuesta de la desalinizadora, debido a sus costos y falta de una visión integral; ha señalado que debería cancelarse por inviabilidad y replantear el contrato (Rubio, 2019).

Igualmente, el manejo de la comunicación del gobierno hacia la sociedad en relación con los alcances del proyecto fue contradictorio en sus declaraciones a la prensa regional. El ejemplo más notable ha sido la información sobre la venta de agua de la planta desalinizadora al Otay Water District, en San Diego. Mientras que diputados opositores se refrieron a información sobre ese propósito del proyecto, los agentes del gobierno de Baja California siempre lo negaron, y trataron de mantener oculta información en tanto la prensa de San Diego publicaba sobre avances en los permisos para que el Otay Water District pudiera comprar agua de la planta en proceso de autorización en Rosarito. Hay que decir al respecto que el proyecto es explícito en cuanto a ese propósito, y en la página oficial de San Diego Water Authority (SDWA) también aparece información sobre las colaboraciones que se han estado llevando a cabo en lo que ellos consideran un proyecto de cooperación binacional.

Por otro lado, información de la oposición política en el congreso local-citando como fuente a la empresa concesionaria- señala que el contrato del gobierno estatal con Aguas de Rosarito SAPI (empresa creada ex profeso para la firma del contrato pero filial de Consolidated Water) habría quedado sin efecto desde el 31 de diciembre de 2018, ante el incumplimiento del primero en su obligación de contratar las garantías financieras para el pago de servicios a la empresa (Heras, 2019). Adicionalmente, el

\footnotetext{
${ }^{11} \mathrm{Al}$ inicio, el principal apoyo del gobierno estatal estuvo en la dirección general de la Conagua de la administración federal anterior, desde donde se impulsaban los proyectos de infraestructura de servicios de agua al amparo de las APP, pero en marzo de 2015 el director general de ese organismo federal, David Korenfeld, renunció a su cargo, con lo cual el gobierno del estado perdió un aliado importante.
} 
proyecto también ha enfrentado los contratiempos de procesos judiciales en torno a la propiedad accionaria entre los socios de la empresa concesionaria, los cuales han tenido que seguir los tiempos procesales de demandas, suspensiones y fallos propios de las demandas aunque, según ejecutivos de la empresa en México, algunas suspensiones han sido retiradas en favor de la empresa (Linaldi, 2019).

\section{El FUtURO DEL PROYECTO dE DESALINIZACIÓN EN ROSARITO}

Con todo lo que ha ocurrido en torno a la desalinizadora en Rosarito, el inicio del proyecto entró en un impasse, pero no parece haber muchas dudas en cuanto a que tarde o temprano se construirá y el agua producida fluirá también hacia San Diego (Mumme et al., 2017). Es casi normal que este tipo de proyectos experimenten retrasos en su ejecución o se pospongan por tiempo indefinido hasta que logran superar los obstáculos. Tomando como referencia la demanda y las condiciones climatológicas de la cuenca mencionadas arriba, todo apunta a fortalecer la idea de que su construcción seguirá adelante. Es importante tener en cuenta que, aunque el término del ciclo político para gobierno del estado que puso en su orden de prioridad la construcción de la planta desalinizadora se ha cerrado, y corresponderá a una nueva administración gubernamental decidir lo procedente respecto a la concesión y los términos de los contratos, es importante tener en cuenta los antecedentes del gobernador y su relación con los intereses que giran en torno del proyecto.

En efecto, Jaime Bonilla, el gobernador del estado a partir de noviembre de 2019, fungió como director del Otay Water District entre los años 2000 y 2011, de modo que es muy difícil negar el vínculo de interés; es decir, desde el punto de vista del proyecto hay una gran posibilidad de continuidad. Al mismo tiempo, algunos de los diputados locales que más se opusieron en el congreso y presentaron el caso en la Suprema Corte de Justicia han sido reelectos y son parte del partido en el gobierno, el cual también gobierna a nivel nacional. Por lo tanto, sólo la desaprobación federal podría frenarlo, aunque es probable que se obligue a negociar los términos de los contratos con la empresa concesionaria.

La concesión para la construcción y operación ya se otorgó y, aunque controvertida, la autorización se ha hecho en las instancias del gobierno y el Poder Legislativo locales; también se han firmado los contratos que comprometen a la empresa y al gobierno del estado. Tratándose de inversiones con origen en Estados Unidos, la cancelación del contrato y la concesión podría ser complicada porque la empresa concesionaria podría apelar invocando el capítulo de protección de las inversiones del Tratado de Libre Comercio trilateral vigente para América del Norte. 
Finalmente, uno de los temas controversiales durante el proceso de aprobación de la concesión fue la posible venta de agua a San Diego. La discusión vertida en medios mostró un manejo de información poco transparente y contradictorio de parte de las autoridades en México, lo que dio lugar a la desconfianza en sectores de la sociedad; sin embargo, vale la pena reflexionar en el marco de las relaciones que se han desarrollado en el contexto binacional y, dentro del mismo, analizar las implicaciones de la venta de agua a la ciudad vecina según los distintos ordenamientos jurídicos que regulan las relaciones bilaterales en materia de agua, comercio y medio ambiente, pero también en un contexto en el que es frecuente hablar de la integración transfronteriza y regional, aunque no hay experiencias internacionales de mercados transfronterizos de agua para abastecer ciudades al otro lado de la frontera internacional.

Visto desde una perspectiva de cooperación hay que tener presente que Tijuana tiene una conexión de emergencia para acceder al agua de San Diego, al amparo de acuerdos renovados en el seno de la cila y desde 2019 ha habido al menos tres envíos de agua solicitados por el organismo operador de Tijuana; se trata de volúmenes que se descuentan de las entregas en Mexicali y, por lo tanto, no forman parte de un mercado transfronterizo de agua. Al mismo tiempo, como lo han señalado Mumme et al. (2017), comercializar el agua en su estado natural a través de la frontera internacional no resulta sencillo debido a las restricciones impuestas por los distintos tratados que rigen las relaciones entre los dos países en el contexto fronterizo. Un tema latente al respecto se refiere a la necesidad de discutir un marco normativo con estándares de calidad ambiental para los dos lados de la frontera, sujetos a la verificación mutua. Su importancia radica en las implicaciones que las plantas desalinizadoras tienen en los distintos tipos de ambientes (marinos, terrestres y atmosféricos), incluido el hecho de que, aun con origen distinto, el agua que se introduzca al sistema de distribución de las ciudades se integra a las cuentas de los volúmenes que de una u otra forma se van a integrar a las cuencas incluidas en el tratado internacional de 1944, así sea como aguas residuales.

\section{Conclusión}

El proyecto aprobado para una planta desalinizadora de agua de mar en Rosarito, en respuesta a la escasez de agua que experimenta la zona costa de Baja California, parece insertarse en la tendencia internacional que ha tenido el desarrollo de este tipo de proyectos en zonas áridas con litoral. Se trata de un proyecto que se ha venido perfilando desde hace tiempo y sobre el cual hay interés en México y en Estados Unidos, lo que da fuerza a la posibilidad de su ejecución; sin embargo, la falta de consenso sobre 
los términos de la concesión y la carga financiera para el gobierno del estado han retrasado el inicio de la construcción y entrada en operación de la desalinizadora que está aprobada y no se ha cancelado. La dependencia tan alta de Tijuana y su área metropolitana del agua del Río Colorado, conducida a través del acueducto desde el Valle de Mexicali situado a ciento ochenta kilómetros, y la condición de escasez impuesta por la climatología en la cuenca de este río sobre el cual hay un decreto de contingencia a partir de agosto de 2019, agregan importancia estratégica a la desalinizadora.

En este punto es importante destacar la pertinencia de las recomendaciones de algunos autores para priorizar la construcción de marcos institucionales que regulen este tipo de proyectos tecnológicos, con objeto de que sean incluyentes y se transparenten los procesos, lo cual evitaría la corrupción y prevendría daños ambientales (McEvoy, 2017). En este caso, la forma en que se concibió el proyecto y se condujo el proceso de aprobación responde al perfil tecnocrático que privilegia la legitimidad técnica y el consenso de élites técnico-financieras sobre las de orden social para la toma de decisiones, es decir, un proceso tecnogerencial tal como lo conceptualizan Syngedouw y Williams (2016), y el resultado hasta ahora está a la vista.

Las discusiones en torno a la desalinizadora han dejado de lado los posibles impactos ambientales y las medidas complementarias de tipo adaptativo para hacer más eficiente el uso del agua (Tijuana y Rosarito tienen los consumos por persona más bajos del país), el reordenamiento de las concesiones de agua en el estado y otras que pudieran discutirse de manera abierta. Cabe señalar que nada se ha dicho sobre mejoras en la eficiencia de la gestión o el tratamiento y posible potabilización de las aguas residuales como ya se hace en varias ciudades del mundo, incluso en la ciudad vecina de San Diego a través del Pure Water Program, que busca disminuir la dependencia de esa ciudad del agua del Río Colorado.

Adicionalmente, la posición del gobierno estatal y la Conagua ha sido ambigua en relación con la posibilidad de exportar agua de la desalinizadora a Otay Mesa en San Diego, cuando allá se considera un hecho, y el plan de negocios de la planta en su versión actual abre una vertiente de relaciones de cooperación transfronteriza a través de la exportación de agua potable, algo inusual a nivel internacional y que, en el caso particular, requiere de reformas legales para facilitar ese tipo de operaciones, aunque es muy probable que se logren acuerdos en ese sentido, considerando los intereses que se mueven en ambos lados de la frontera en torno al proyecto. 


\section{FUENTES}

Andrés, María de y Juan Manuel Barragán

2016 "Desarrollo urbano en el litoral a escala mundial. Método de estudio para su cuantificación", Revista de Estudios Andaluces, vol. 33, no. 1, pp. 64-83.

Arahuetes, Ana y Rubén Villar

2017 "Desalination, Strategic and Controversial" (conference paper), wIT Transactions on Ecology and The Environment, vol. 216, pp. 61-72, DOI: 10.2495/WS170061.

Arellano SARMiento, Luis

2017 “Desalinizadoras APP están 'palomeadas' por el gobierno federal: Vega", La Jornadab, 22 de septiembre, en <http://jornadabc.mx/tijuana/22-09-2017/ desalinizadoras-app-estan-palomeadas-por-el-gobierno-federal-vega $>$, consultada el 5 de marzo de 2018.

BerkTAy, Alí

2011 "Environmental Approach and Influence of Red Tide to Desalination Process in the Middle East Region", International Journal of Chemical and Environmental Engineering, vol. 2, pp. 183-188.

Bernardo, Valeria, Xavier Fageda y Montserrat Termes

2015 "Do Droughts Have Long-term Effects on Water Consumption? Evidence from the Urban Area of Barcelona", Applied Economics, nos. 47 y 48, pp. 5131-5146, doi: $10.1080 / 00036846.2015 .1042147$.

Bustamante Redondo, JoAQuín

1999 La Comisión Internacional de Limites y Aguas entre México y los Estados Unidos, Ciudad Juárez, Universidad Autónoma de Ciudad Juárez, 605 pp.

Castro, José Luis y Vicente Sánchez

2008 "Water Management in the San Diego-Tijuana Region: What Lessons Can Be Learned?", en James Loucky, Donald K. Alper y John C. Day, eds., Policy Challenges in the Pacific Regions of North America Transboundary, Calgary, Al., University of Calgary Press, pp.171-193. 


\section{City OF SAN DiEgo}

s. f. "Pure Water San Diego", en <https: / /www.sandiego.gov/public-utilities / sustainability/ pure-water-sd>, consultada el 28 de mayo de 2020.

\section{Comisión Estatal de Servicios Públicos de Tijuana (cespt)}

2017 "Resumen gerencial de datos básicos e indicadores de gestión", en <https: / / www. cespt.gob.mx/Documentos/Transparencia / Art_70/70_30/30_01_2017_BASI COSINDICADORESGESTION.pdf >, consultada el 15 de noviembre de 2018.

\section{Comisión Estatal del Agua (cea)}

2015 "Descripción y viabilidad técnica del Proyecto", en <http:/ / www.ceabc. gob.mx / documents / transparencia / NUEVA\%20PLATAFORMA / DESAL ROS / 1.Descripci\%C3\%B3n\%20y\%20Viabilidad\%20T\%C3\%A9cnica-ROS. pdf $>$, consultada el 12 de mayo de 2018 .

s. f. "Acueducto Río Colorado", en <http: / www.cea.gob.mx/arct.html>, consultada el 12 de mayo de 2018.

\section{COMisión de Límites y AguAs (CILA)}

2012 Acta 319 “Medidas interinas de cooperación internacional en la Cuenca del Río Colorado hasta el 2017 y ampliación de las medidas de cooperación del Acta 318, para atender los prolongados efectos de los sismos de abril de 2010 en el Valle de Mexicali, Baja California", en <http: / / www.cila.gob.mx/actas/319. pdf $>$, consultada el 5 de enero de 2018.

\section{Comisión Nacional del Agua (Conagua)}

2017 "Proyectos estratégicos, de agua potable, drenaje y saneamiento" (Presentación), en <https: / /www.gob.mx/cms/uploads / attachment/file/187125/ Estrategicos-enero-2017.pdf $>$, consultada el 15 de enero de 2018.

2015 "Proyectos estratégicos, de agua potable, drenaje y saneamiento" (Presentación), en <https://www.gob.mx/conagua/documentos/proyectos-estrate gicos-28811>, consultada el 15 de enero de 2018.

2012 "Proyectos estratégicos, de agua potable, drenaje y saneamiento" (Presentación), en <https://agua.org.mx/wp-content/uploads/2012/07/proyectoses trategicosaguapotabledrenajesaneamiento_conagua2012.pdf $>$, consultada el 5 de enero de 2018. 


\section{CONSOlidated WATER}

2015 "Rosarito Project-Mexico", en <http: / / cwco.com/portfolio/mexico / >, consultada el 4 de abril de 2018.

Corpus, Aline

2018 "Van diputados contra la desaladora en BC", Reforma, en < https: / / www.re forma.com/ aplicacioneslibre / articulo/ default.aspx?id=1291738\&md5=f9b 793086ded1a789c48f6c932669a7b\&ta=0dfdbac11765226904c1\%E2\%80\%A6>, consultada el 2 de septiembre de 2019.

\section{Correa Díaz, Felipe}

2007 “Evaluación de la sustentabilidad en la instalación de plantas desaladoras de agua de mar en la región noroeste de México", tesis doctoral, Departamento de Ingeniería de Procesos, Universidad de Las Palmas de Gran Canaria, julio, en <http:/ / oceanologia.ens.uabc.mx/ felipecorrea/ulpgc/introC.htm>, consultada el 28 de enero de 2019.

\section{Cosín, CARLOS}

2019 "The Evolution Rates in Desalination (Part I)", en <https:/ / smartwaterma gazine.com/blogs/ carlos-cosin/ evolution-rates-desalination-part-i $>$, consultada el 30 de junio de 2019.

\section{Dawoud, Mohamed A. y Mohamed M. Al Mulla}

2012 "Environmental Impacts of Seawater Desalination: Arabian Gulf Case Study", International Journal of Environment and Sustainability, vol. 1, pp. 22-37.

Drami, Dror, Yosef Z. Yacobi, Noga Stambler y Nurit Kress

2011 "Seawater Quality and Microbial Communities at a Desalination Plant Marine Outfall. A Field Study at the Israeli Mediterranean Coast", Water Research no. 45 , pp. $5449-5462$.

El Saliby, Ibrahim, Yousef H. OKour, Ho Kyong Shon y Kim In S.

2008 "Desalination Plants in Australia. Review and Facts", Desalination, no. 247, pp. 1-14.

FiKes, BRAdLEY, J.

2015 "Inauguran planta desalinizadora", San Diego Union Tribune, en <http:/ / www.sandiegouniontribune.com/hoy-san-diego/sdhoy-inauguran-plantadesalinizadora-2015dec25-story.html >, consultada el 2 de agosto de 2018. 
García, Esperança, Olga Invers, Marta Manzanera, ENRIC BALLESTEROS Y JAVIER ROMERO

2007 "Impact of the Brine from a Desalination Plant on a Shallow Seagrass (Posidonia oceanica) Meadow", Science Direct, no. 72, pp. 579-590.

García-Rubio, Miguel Angel y Jorge Guardiola

2012 "Desalination in Spain: A Growing Alternative for Water Supply", International Journal of Water Resources Development, vol. 28, no.1, pp. 171-186, DOI: 10.1080 / 07900627.2012.642245.

Gobierno de Baja CALifornia (GBC)

s.f. <http:/ / www.bajacalifornia.gob.mx/portal/nuestro_estado/recursos/hi drologia.jsp $>$, consultada el 15 de junio de 2019.

Hartley, Kris, Cecilia Tortajada y Asit K. Biswas

2019 "A Formal Model Concerning Policy Strategies to Build Public Acceptance of Potable Water Reuse", Journal of Environmental Management, no. 250, pp. 1-11.

Heras, ANTONIO

2019 "Desaladora en BC sin respaldo financiero del gobierno", La Jornada Baja California, en <http:/ /jornadabc.mx/tijuana/13-01-2019/ desaladora-en-bc-sinrespaldo-financiero-del-gobierno>, consultada el 4 de abril de 2019.

HÖPNER, THOMAS Y JENS WiNDELBERG

1996 "Elements of Environmental Impact Studies on Coastal Desalination Plants", Desalination, no. 108, pp. 11-18.

Lattemann, Sabine y Thomas HöPner

2008 "Environmental Impact and Impact Assessment of Seawater Desalination", Estuarine Costal and Shelf Science, vol. 72, no. 220, pp. 1-15.

Lattemann, Sabine, María D. Kennedy, Jan C. Schippers y Amy Gary

2010 “Global Desalination Situation", Elsevier vol. 2, cap. 2, pp. 7-39.

LiNALDI, CARLOS

2019 "Desaladora de Rosarito, va: empresa", Noticias, en <https:/ / cadenanoti cias.com/regional/2019/08/ desaladora-de-rosarito-va-empresa >, consultada el 10 de septiembre de 2019. 


\section{LóPEZ, EDUARDO}

2019a "La planta desaladora, tema que se partidizó: López Nogales", El Imparcial, en <https: / www.elimparcial.com/sonora/ sonora/La-planta-desaladora-temaque-se-partidizo-Lopez-Nogales-20190329-0094.html>, consultada el 20 de agosto de 2019.

2019 b "Desaladora fue un tema de pesos y centavos: Búrquez", El Imparcial, en <https: / / www.elimparcial.com/ sonora/ hermosillo/Desaladora-fue-un-temade-pesos-y-centavos-Burquez-20190330-0098.html>, consultada el 20 de agosto de 2019.

Loya-Fernández, Ángel, Luis Miguel Ferrero-Vicente, Candela Marco-Méndez, Elena MartíneZ-García, José Zubcoff y José Luis SánCheZ-Lizaso

2012 "Comparing Four Mixing Zone Models with Brine Discharge Measurements from a Reverse Osmosis Desalination Plant in Spain", Desalination, no. 286, pp. 217-224.

McEvor, JAMIE

2017 "Can the Adoption of Desalination Technology Lead to Aquifer Preservation? A Case Study of a Sociotechnical Water System in Baja California Sur, Mexico", Water, vol. 7, no. 10, pp. 5224-5238.

Meerganz von Medeazza, G.L.

2005 "'Direct' and Socially-induced Environmental Impacts of Desalination", Desalination, no. 185 , pp. 57-70.

Morote, Álvaro-Francisco, Antonio-Manuel Rico y Enrigue Moltó

2017 "Critical Review of Desalination in Spain: A Resource for the Future?", Geographical Research, vol. 55, no. 4, pp. 1-12.

Mumme, Stephen P., Jamie McEvoy, Nicolas Pineda y Margaret Wilder

2017 "Shipping Water Across the US-Mexico Border: International Governance Dimensions of Desalination for Export", Water International, DOI: 10.1080/ 02508060.2017 .1373320 .

Nair, Majula y Dinesh Kumar

2013 "Water Desalination and Challenges: The Middle East Perspective. A Review", Desalination and Water Treatment, vol. 51, nos. 10-12, pp. 2030-2040, DOI: 10.1080 / 19443994.2013.734483. 
NegRete, José

1990 “Agua y conflicto en Tijuana 1979-1990" (ponencia), Primer Seminario Interno de El Colegio de la Frontera Norte, Tijuana, B.C.

Pombo, Alberto

2015 "Adaptaciones tecnológicas en el manejo del agua y sus consecuencias en la población de la región agrícola de San Quintín, Baja California", en Hugo Riemann, coord., El agua en la región agrícola de Camalú-El Rosario, Baja California. Un recurso sobreexplotado con repercusiones sociales y ambientales, Puebla, Red Nacional de Investigación Urbana, pp. 81-94.

Pombo, Alberto, Aurora Breceda y Alba Valdez Aragón

2008 "Desalinization and Wastewater Reuse as Technological Alternatives in an Arid, Tourism Booming Region of Mexico", Frontera Norte, vol. 20, no. 39, pp. 7-32.

Rubio, DANIEL ÁNGEL

2019 "Desaladora no es viable, dice Osuna", El Sol de Tijuana, en <https: / www. elsoldetijuana.com.mx / local / desaladora-no-es-viable-dice-osuna-3371132. html>, consultada el 10 de octubre de 2019.

SÁnchez-Munguía, Vicente y Alfonso A. Cortez-Lara

2015 "Minute 319 of the International Boundary and Water Commission between the U.S. and Mexico. Colorado River Binational Water Management Implications", International Journal of Water Resources Development, vol. 31, no. 1, pp. 17-27.

SWYNGEDOUW, ERIK y Joe WiLliams

2016 "From Spain's Hydro-deadlock to the Desalination Fix", Water International, vol. 41 , no. 1 , pp. 54-73.

TAL, ALON

2017 "The Evolution of Israeli Water Management: The Elusive Search for Environmental Security", en Jean Axelrod Cahan, ed., Water Security in the Middle East, Nueva York, Anthem Press, pp. 125-143.

2011 "The Desalination Debate-Lessons Learned Thus Far, Environment", Science and Policy for Sustainable Development, vol. 53, no. 5, pp. 34-48, Dor: doi.org/ 10.1080/00139157.2011.604009. 
VOUTCHKOV, NiKOLAY

2016 "Desalination-Past, Present and Future", en http:/ / iwa-network.org/author/ nikolay-voutchkov, consultada el 30 de noviembre de 2018.

Wilder, Margaret O., Ismael Aguilar-Barajas, Nicolas Pineda-Pablos,

Robert G. Varady, Sharon B. Megdal, Jamie McEvoy, Robert Merideth, Adriana ZúÑIga-Terán y CHRISTOPHer A. SCOTT

2016 "Desalination and Water Security in the US-Mexico Border Region: Assessing the Social, Environmental and Political Impacts", Water International, vol. 41, no. 5, pp. 756-775. 